\title{
AAV2-mediated gene transfer of VEGF-Trap with potent suppression of primary breast tumor growth and spontaneous pulmonary metastases by long-term expression
}

\author{
LIAN LU*, SHUN-TAO LUO*, HUA SHAN SHI, MENG LI, HAI LONG ZHANG, \\ SHA SHA HE, YAN LIU, YING PAN and LI YANG
}

State Key Laboratory of Biotherapy and Cancer Center, West China Hospital, West China Medical School, Sichuan University, Sichuan 610041, P.R. China

Received March 7, 2012; Accepted April 17, 2012

DOI: 10.3892/or.2012.1915

\begin{abstract}
Vascular endothelial growth factor (VEGF) is an important signaling protein and a predominant mediator of angiogenesis in tumor growth and metastasis. Therefore, antagonism of the VEGF pathway results in inhibition of abnormal angiogenesis, then suppression of tumor growth and metastasis. VEGF-Trap, a high-affinity soluble decoy receptor, is currently in phase II clinical trails, and has demonstrated more efficacy in different types of solid tumors by intravenous injection every two weeks. In our study, we used recombinant AAV2 as a delivery vehicle to achieve long-lasting expression of VEGF Trap protein in a mouse model for the first time. We report that AAV2VEGF-Trap can be safely administered and sustained expression in vivo via a single intravenously administration, simultaneously suppressing primary tumor growth and preventing the pulmonary metastases of 4T1 tumors. Decreased microvessel density and increased tumor cell apoptosis were observed in the treatment group. AAV2-VEGF-Trap can obviously decrease not only the concentration of VEGF in sera, but also the concentration of other angiogenic factors, such as aFGF, bFGF, angiopoietin-1 and others. These studies suggest that AAV-mediated long-term expression of VEGF-Trap is a useful and safe tool to block tumor progression and inhibit spontaneous pulmonary metastases.
\end{abstract}

\section{Introduction}

Angiogenesis is necessary for tumor growth, invasiveness and development of metastasis (1-4). Angiogenesis is a complex process that is tightly regulated by pro- and anti-angiogenic

Correspondence to: Professor Li Yang, State Key Laboratory of Biotherapy, West China Hospital, Sichuan University, Keyuan Road 4, Chengdu, Sichuan 610041, P.R. China

E-mail: yl.tracy73@gmail.com

*Contributed equally

Key words: tumor, metastasis, AAV2, VEGF-Trap, long-term expression growth factors (5). At present, it is clear that the role of angiogenic factors include VEGF, aFGF, bFGF, angiogenin, PD-ECGF, TGF and TNF (6-9). The predominant regulator of tumor angiogenesis is vascular endothelial growth factor (VEGF) (10-12), which plays a vital role during normal embryonic angiogenesis, endothelial cell proliferation and also in the physiological and pathological angiogenesis. Furthermore, an essential role for VEGF in tumor angiogenesis has been demonstrated in an animal model (13). VEGF has received attention as a target for therapeutic angiogenesis (14).

VEGF-targeted therapies were generally neutralizing VEGF antibody and dominant-negative VEGF receptors (15). A previous study has described antiangiogenic therapy with VEGF-Trap that may be a new and perhaps more effective approach to block tumor-associated VEGF, and act as a soluble decoy receptor and a high-affinity anti-VEGF agent for VEGF. VEGF-Trap consists of portions of the extracellular domains of both VEGFR-1 and VEGFR-2 fused to an Fc segment of human $\operatorname{IgG}$ (16). VEGF-Trap, an antiangiogenic agent, can prevent VEGF to bind to its receptor, in order to block activities of VEGF. Suppressing vascularization and tumor growth in vivo by VEGF-Trap, tumors appears to be stunted and almost completely avascular. The high affinity fusion proteins have been reported to cause regression of coopted vessels, therefore suppressing tumor growth by inhibiting tumor induced angiogenesis (17). A recently study demonstrated that VEGF-Trap treatment was efficacious in both initial and advanced phases of tumor development by significantly prolonging survival (18). In order to obtain long-term tumor-free survival by VEGF-Trap therapy, the agent inhibiting tumor neovasculature needs to be systemically maintained at stable therapeutic levels. Administration of purified VEGF-Trap needs multiple administrations and may be limited by their short half-life with associated discomfort and cost $(4.0 \mathrm{mg} / \mathrm{kg}$ intravenously injection every two weeks in clinical trails). Thus, production of VEGF-Trap may overcome these limitations after gene transfer.

There are some requirements for vectors used in antiangiogenic gene therapy, for example, no or low toxicity or immunity, and the sustained expression. Therefore, adeno-associated virus (AAV) is a very attractive candidate for creating viral 
vectors for gene therapy (19-21). Adeno-associated virus (AAV) as vectors are non-pathogenic and less immunogenic compared with other gene therapy vectors (22). To date, AAV vectors have been used for many clinical trials for treatment of certain tumors (23). The present study evaluated recombinant AAV (rAAV) encoding secretable forms of VEGF-Trap. We show that a single intravenous administration of adeno-associated virus-mediated gene therapy expressing VEGF-Trap resulted in persistent expression of VEGF-Trap, reduction of the concentration of VEGF and other antigenic factors, such as aFGF, bFGF, angiogenin and PD-ECGF, inhibition of tumor growth and prevention of lung metastases formation in 4T1 metastatic breast cancer model.

\section{Materials and methods}

Cell lines. The murine breast carcinoma $4 \mathrm{~T} 1$ cells were purchased from the American Type Culture Collection (ATCC; Rockville, MD, USA). These cells were cultured in RPMI-1640 supplemented with $10 \%$ fetal bovine serum, $2 \mathrm{mM}$ glutamine, and antibiotics $(100 \mathrm{U} / \mathrm{ml}$ penicillin and $100 \mu \mathrm{g} / \mathrm{ml}$ streptomycin). Cells were grown at $37^{\circ} \mathrm{C}$ in a humidified $5 \%$ $\mathrm{CO}_{2}$ atmosphere.

Western blot analysis of AAV2-VEGF-Trap in vitro. AAV-VEGF-Trap and AAV-Null virus were transfected into HEK293 cells (ATCC; Rockville). After 48 h, the media of tranfected 293 cells were collected, concentrated and electrophoresed in 12\% SDS-PAGE. Then, proteins were transferred at $100 \mathrm{~V}$ for $1 \mathrm{~h}$ to a polyvinylidene difluoride (PVDF) membrane (Bio-Rad, Richmond, CA, USA). The membranes were blocked with $5 \%$ milk in TBST at $4^{\circ} \mathrm{C}$ overnight, then incubated with either human VEGFR1/Flt-1 antibody (R\&D Systems, USA) or human VEGFR2/KDR antibody (R\&D Systems) for $1 \mathrm{~h}$ at $37^{\circ} \mathrm{C}$. Membranes were washed three times in TBST and HRP-conjugate rabbit anti-goat immunoglobulins (R\&D Systems) were incubated. The protein bands were visualized via an enhanced chemiluminescence detection system (Pierce, Rockford, IL, USA).

Breast carcinoma model. Female Balb/c mice, 6 weeks of age, were purchased from Beijing Hua Fu Kang Biological Technology Co.,Ltd. All animals were housed under pathogenfree conditions and fed autoclaved pellets and water. Mice were inoculated s.c. with $5 \times 10^{5} 4 \mathrm{~T} 1$ tumor cells. After small s.c. tumors became about $3 \mathrm{~mm}$ (about 1 week after implantation), treatment with the AAV2-VEGF-Trap was initiated. The mice were divided into three groups after inoculated s.c. with 4T1 tumor cells and each group had six animals. Seven days after inoculation, AAV2-VEGF-Trap or AAV2-Null in $50 \mu 1$ of PBS was administered intravenously. The first group was injected with normal saline (NS). The second group of animals was treated with $6 \times 10^{9}$ viral genomes (vg) AAV2VEGF-Trap, and the third group with $6 \times 10^{9} \mathrm{vg}$ of AAV2-Null. Tumor size was measured every 3 days with a digital caliper for two-dimensional length and width, and tumor volume was calculated as (length $\mathrm{x}$ width $\left.{ }^{2}\right) / 2$.

Two groups of normal female Balb/c mice, 6 weeks of age, were intravenously administered with AAV2-VEGF-Trap $\left(6 \times 10^{9} \mathrm{vg}\right)$ or AAV2-Null $\left(6 \times 10^{9} \mathrm{vg}\right)$. The sera were collected every week for 13 weeks to measure the levels of VEGF Trap by ELISA.

Expression of VEGF-Trap by ELISA. Levels of VEGF-Trap were measured by ELISA. Briefly, blood was collected through the tail vein on days $0,1,3,7,21$ and 28 after AAV2-VEGFTrap administration in the tumor model and the sera were collected every week for 13 weeks in two groups of normal female Balb/c mice. ELISA plates were coated with $2 \mu \mathrm{g} / \mathrm{ml}$ hVEGF165 in carbonate/bicarbonate buffer at $4^{\circ} \mathrm{C}$ overnight. Plates were washed three times with PBST $(0.1 \%$ Tween in PBS) and blocked with $0.5 \%$ solution of non-fat dried milk in PBST for $1 \mathrm{~h}$ at $37^{\circ} \mathrm{C}$. Serum samples were diluted in $1: 5$ and added to the wells, then and incubated for $1 \mathrm{~h}$ at $37^{\circ} \mathrm{C}$. Later, plates were incubated with $1 \mu \mathrm{g} / \mathrm{ml}$ of human VEGFR1/Flt-1 biotinylated antibody (R\&D Systems) at $37^{\circ} \mathrm{C}$ of $1 \mathrm{~h}$. After washed, the secondary antibody was Streptavidin-HRP (R\&D Systems) diluted 1:200. After five washes with PBST, the substrate was developed with 3,3',5,5'-tetramethyl benzidine (TMB) (Sigma-Aldrich, USA), which was developed at RT for 20 min and stopped with $2 \mathrm{M} \mathrm{H}_{2} \mathrm{SO}_{4}$. Plates were read by a Bio-Rad microplate reader at $450 \mathrm{~nm}$.

Immunohistochemistry. The tissues were fixed in $4 \%$ paraformaldehyde and embedded in paraffin. Lungs and tumors were cut into sections and stained with hematoxylin and eosin. Frozen tumor tissues were stained for blood vessels using a monoclonal rabbit anti-mouse CD-31phycoerythrin conjugate (BD, USA). Nuclei were stained with 4',6-diamidino-2-phenylindole (DAPI; dilution 1:5000). Apoptosis was determined by TUNEL staining, using DeadEnd $^{\mathrm{TM}}$ Fluorometric TUNEL System (Promega, USA). The sections were examined with an upright fluorescence microscope. In addition, tissues simples of kidney, liver, heart and brain were sectioned and stained with $\mathrm{H} \& \mathrm{E}$ to measure the toxicity.

Analyzing angiogenesis-related proteins levels in the serum. To examine the change of angiogenesis-related protein levels, serum was collected and stored at $-20^{\circ} \mathrm{C}$. Mouse Angiogenesis Array kit (R\&D System), which can detect expression levels of 53 mouse angiogenesis related proteins, was used. Array data can be quantified on developed X-ray film by scanning the film on transmission-mode and using image analysis software (Quantity One) to analyze the array image file.

Statistical analysis. SPSS 17.0 was used for statistical analysis. Data were expressed as mean \pm SE. One-way analysis of variance or the unpaired Student's t-test were used for comparison between groups. Differences were considered statistically significant at $\mathrm{P}<0.05$. Comparisons of image analysis measurement, tumor volume and metastasis were performed.

\section{Results}

Inhibition of s.c. tumor growth and spontaneous lung metastases. VEGF is necessary for the development of neovasculatures at very early stages of tumorigenesis (24-26). AAV2-VEGF-Trap was injected intravenously at early stage of tumor about $3 \mathrm{~mm}$ in diameter. Although, every animal 
A

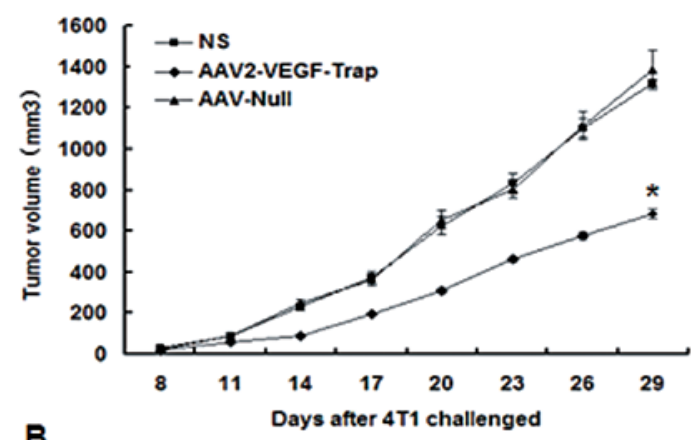

B

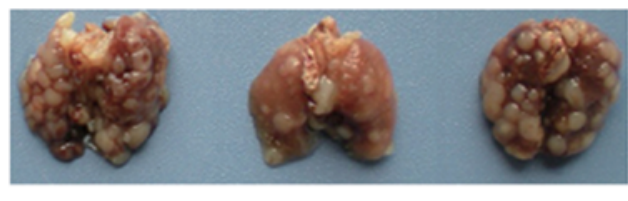

NS

AAV2-VEGF-Trap

AAV2-Null

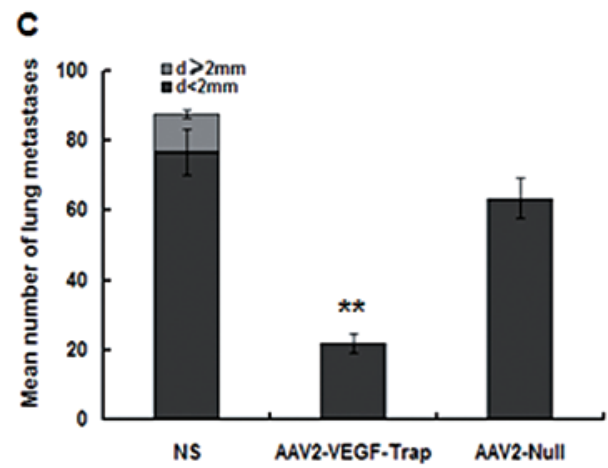

D

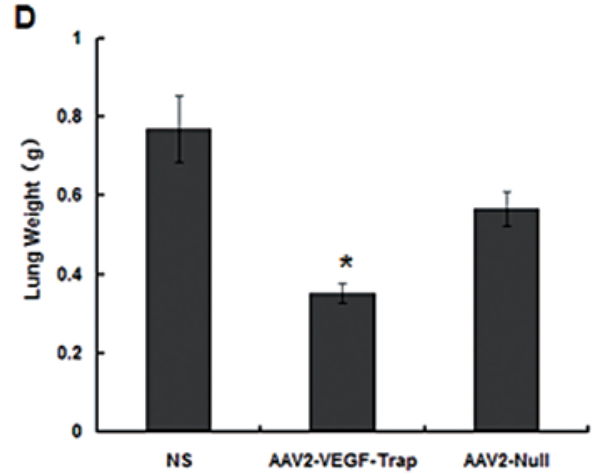

Figure 1. Inhibition of tumor growth and pulmonary metastases after initiation of AAV2-VEGF-Trap injections. The mice were challenged s.c. with $5 \times 10^{5} 4 \mathrm{~T} 1$ cells. After 7 days, the first group of mice were treated with normal saline, the second group was treated with AAV2-VEGF-Trap (6x10 ${ }^{9}$ viral genomes), and the third group of mice were treated with AAV2-Null (6x $10^{9}$ viral genomes). (A) The tumor size on each mouse was monitored every 3 days. There was a great difference in tumor volume between the experiment group (AAV2-VEGF-Trap) and the control groups (NS, AAV2-Null). (B) Surface of lung metastases. (C) The number of lung metastases. (D) Lung weight. ( $\mathrm{P}<0.05,{ }^{* *} \mathrm{P}<0.001$ AAV2-VEGF-Trap vs. AAV2-Null and NS).

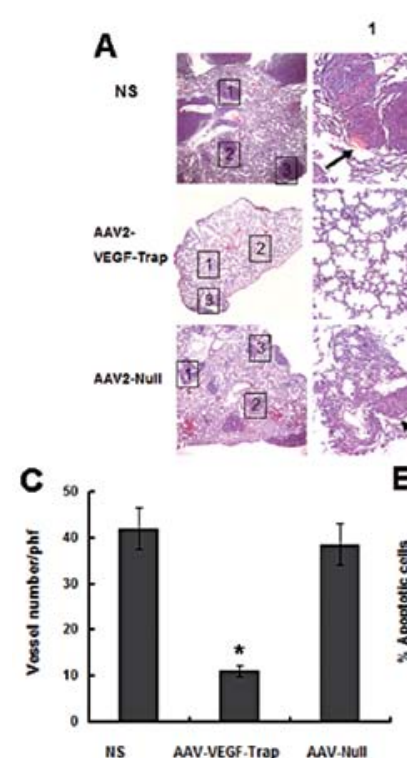

MS AAV-VEGF-Trap AAV-Mull
12

23

3

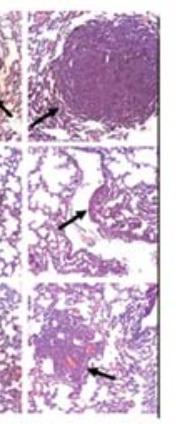

E

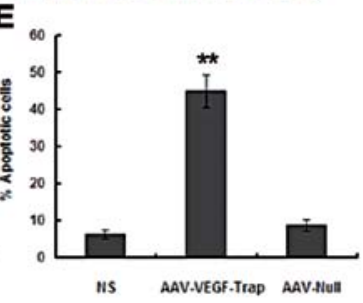

B

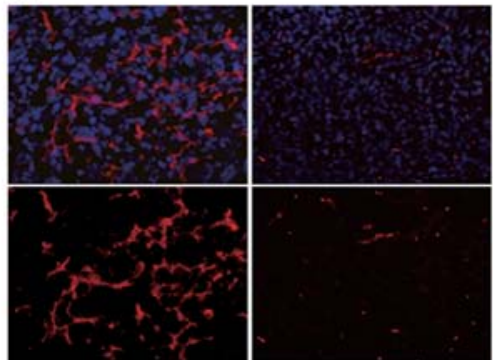

AAV2-VEGF-Trap

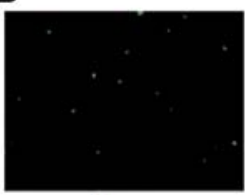

NS

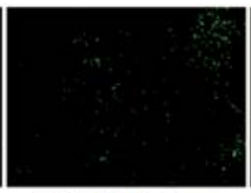

AAV2-VEGF-Trap

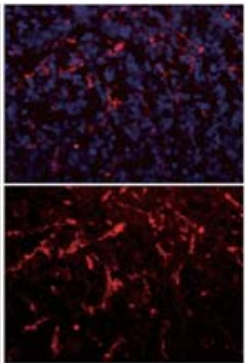

AAV2-Null

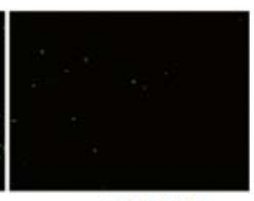

AAV2-Null

Figure 2. Inhibition of tumor angiogenesis within tumor tissue stained by CD31 and detection of apoptosis by TUNEL staining of tumor tissue. (A) Lung sections stained with H\&E. Three areas of the lung sections (indicated as black lined squares) were observed under high magnification and the arrows indicate clustered metastatic tumors (magnification, x200). (B) Top, section of frozen 4T1 tumor tissue stained with CD31 and DAPI. Bottom, vessel staining with CD31 vascular density was determined by counting the number of the microvessels per high-power field (magnification, $\mathrm{x} 400)$. (C) Densities of CD31 ${ }^{+}$blood vessel in tumor. Apoptotic tumor cells were visualized and quantified using the TUNEL assay. (D) TUNEL staining in each group (magnification, $\mathrm{x} 200$ ); (E) quantitation by the TUNEL assay. $\left({ }^{*} \mathrm{P}<0.05,{ }^{* *} \mathrm{P}<0.001\right.$ AAV2-VEGF-Trap vs. AAV2-Null and NS).

developed a tumor, the growth rate of the AAV2-VEGF-Trap group was significantly slower than that in the NS group and AAV2-Null group (Fig. 1A). The mice of all groups were chal- lenged with $5 \times 10^{5} 4 \mathrm{~T} 1$ breast tumor cells by s.c. inoculation. 4T1 tumor cells can trigger spontaneous lung metastases about $1.5 \mathrm{~cm}$. Comparison of the respective metastatic surface areas 


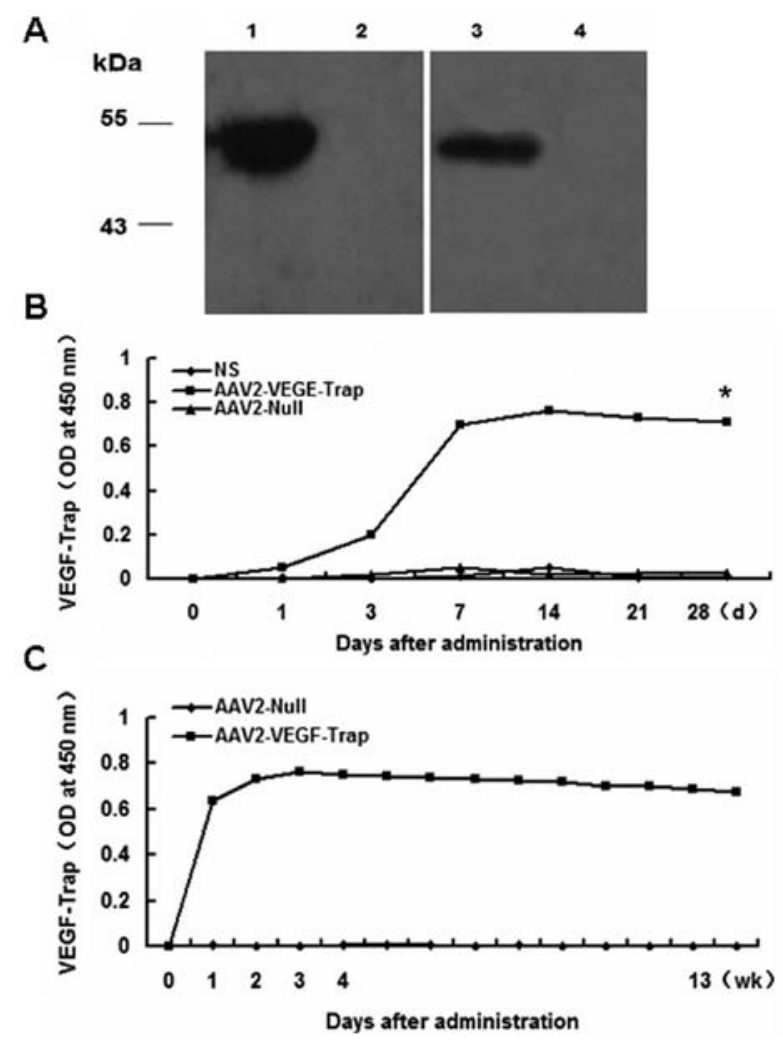

Figure 3. Expression of AAV2-VEGF-Trap. In vitro, western blot analysis expression of VEGF-Trap from HEK293 cells transfected AAV2-VEGF-trap and long-term expression of AAV2-VEGF-Trap in serum. Blood was subsequently obtained on days $0,1,3,7,14,21$ and 28 in the mice with $4 \mathrm{~T} 1$ tumor and obtained for 13 weeks in normal mice. Then, the sera were analyzed by ELISA. (A) Western blot analysis of the expression of VEGF-Trap using anti-hVEGFR1/ flt-1 (lane 1, AAV2-VEGF-Trap; lane 2, AAV2-Null), anti-hVEGFR2/KDR (lane 3, AAV2-VEGF-Trap; lane 4, AAV2-Null). (B) Sustained expression of AAV2-VEGF-Trap for 28 days in the 4T1 model. (C) Sustained expression of AAV2-VEGF-Trap for 13 weeks in normal mice injected AAV2-VEGF-Trap. ('P<0.05 AAV2-VEGF-Trap vs. AAV2-Null and NS).

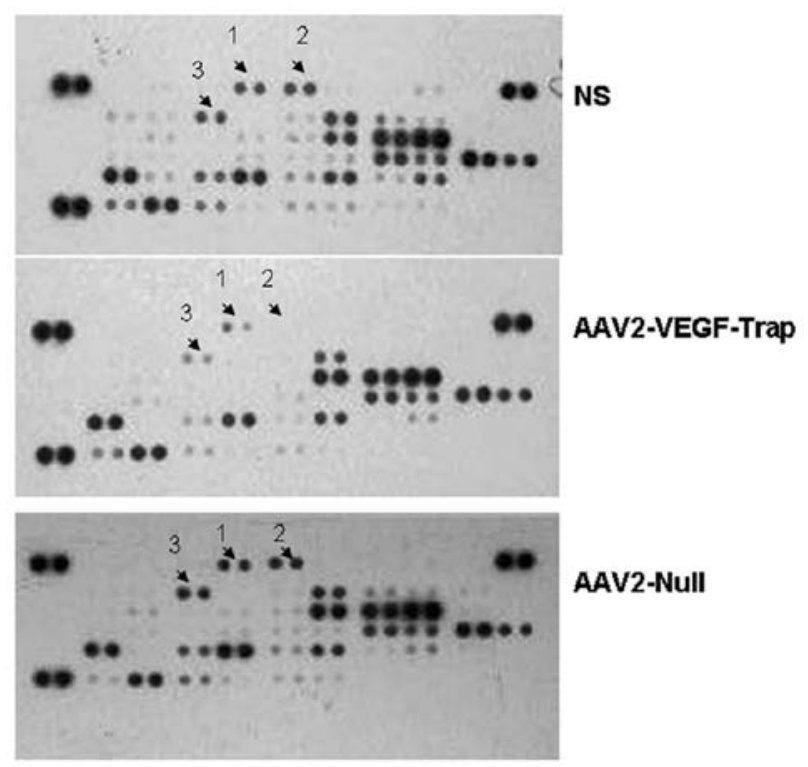

Figure 4. AAV2-VEGF-Trap affected the expression of angiogenesis-related factors. The Mouse Angiogenesis Array detects multiple analytes in serum sample. Profiles of mean spot pixel density were created using a transmission-mode scanner and image analysis software. (A) Membrane images are shown (1, angiogenin; 2, angiopoietin; 3, DPPIV).

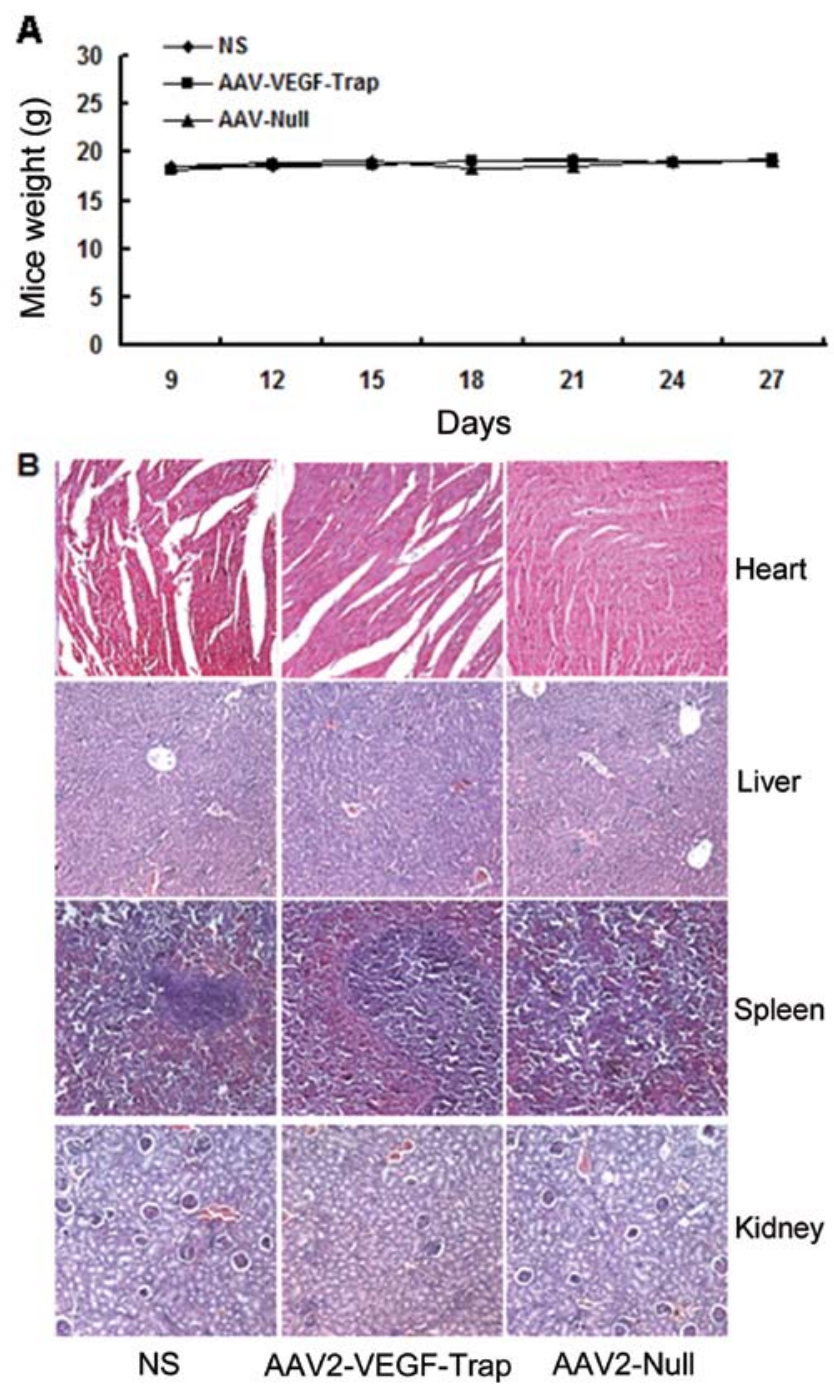

Figure 5. Body weight and sections of major organs. Body weight was measured every 3 days and on Day 28 after treatment, major organs were sampled. (A) Body weight. (B) Sections of heart, liver, spleen and kidney were stained with $\mathrm{H} \& \mathrm{E}$ among the three groups. No obvious difference in each group (magnification, $\mathrm{x} 200$ ).

showed that AAV2-VEGF-Trap group significantly reduced metastatic load, compared to the other groups (Fig. 1B and C).

However, pulmonary tumor deposits were strikingly smaller in the AAV2-VEGF-Trap-treated lungs in comparison with the controls (Fig. 2A). At the same time, the lung weight of AAV-VEGF-Trap group was obviously less than that of NS group and AAV2-Null group (Fig. 1D). These results indicated that AAV2-VEGF-Trap can effectively inhibit the growth of primary breast tumors and spontaneous pulmonary metastases in mice with established 4T1 breast tumor.

Inhibition of angiogenesis and promotion of tumor apoptosis. To further investigate whether the decreased tumor growth was associated with a reduction in the tumor angiogenesis, frozen tumor sections were stained with anti-CD31 antibody (Fig. 2B, lower) and counterstained with DAPI (Fig. 2B, upper). The AAV2-VEGF-Trap group exhibited apparent inhibition of angiogenesis compared with the control groups (the NS group and AAV2-VEGF-Trap group) (Fig. 2C). In order to estimate 
Table I. Mean pixel density of angiogenesis factors of each group.

\begin{tabular}{|c|c|c|c|c|}
\hline & & \multicolumn{3}{|c|}{ Mean pixel density } \\
\hline & & NS & AAV2-VEGF-Trap & AAV2-Null \\
\hline 1 & Angiopoietin-3 & 23285.42743 & 0 & 54752.14071 \\
\hline 2 & CXCL16 & 47495.42495 & 0 & 127174.0433 \\
\hline 3 & Cyr61 & 51711.83539 & 0 & 50608.70028 \\
\hline 4 & DLL4 & 36233.71378 & 0 & 42994.55948 \\
\hline 5 & DPPIV & 105744.736 & 67509.88204 & 105357.301 \\
\hline 6 & Endothelin-1 & 67219.70701 & 0 & 78493.54819 \\
\hline 7 & FGF acidic & 43989.51959 & 0 & 67765.62207 \\
\hline 8 & FGF basic & 49741.49019 & 0 & 37905.64895 \\
\hline 9 & Fractalkine & 39346.7841 & 0 & 58738.60613 \\
\hline 10 & HB-EGF & 34086.33856 & 0 & 54464.60068 \\
\hline 11 & $\mathrm{HGF}$ & 43835.77957 & 0 & 53129.75554 \\
\hline 12 & Leptin & 32774.45842 & 0 & 53819.95561 \\
\hline 13 & PlGF-2 & 45577.01475 & 0 & 41604.89934 \\
\hline 14 & Prolactin & 109857.0365 & 67141.787 & 91828.23958 \\
\hline 15 & PDGF-AA & 87302.40411 & 57683.83602 & 78166.29315 \\
\hline 16 & Serpin F1 & 72810.73259 & 52744.2355 & 77245.32806 \\
\hline 17 & Endoglin & 36370.85379 & 0 & 59343.50619 \\
\hline 18 & Angiogenin & 103220.4658 & 64170.16169 & 160876.2768 \\
\hline 19 & IGFBP-3 & 349666.8265 & 43272.03951 & 397592.7965 \\
\hline & Angiopoietin-1 & 118544.2774 & 0 & 181185.0739 \\
\hline
\end{tabular}

apoptosis in tumor tissues, TUNEL staining was performed. Apparently more apoptotic cells were observed in the tumor sections of mice treated with AAV2-VEGF-Trap than that with AAV2-Null and NS (Fig. 2D and E).

Expression of AAV2-VEGF-Trap in vitro and long-term expression in vivo. The above studies indicated that AAV2VEGF-Trap could block the formation of neovascularization and inhibit tumor growth, due to the expression of VEGF-Trap. In vitro, western blot analysis of the cell supernatant revealed the expression of VEGF-Trap following transfected with AAV2VEGF-Trap comparing with AAV2-Null (Fig. 3A). Next, we monitored the expression of VEGF-Trap in the sera of mice injected rAAV2-VEGF-Trap in the tumor model. The blood of the mice was obtained on days $0,1,3,7,21$ and 28 after intravenous administration of $6 \times 10^{9} \mathrm{vg}$ of AAV2-VEGF-Trap; and the sera were used to analyze VEGF-Trap by ELISA. VEGFTrap levels remained at similar levels until the last evaluation at 28 days (Fig. 3B). No VEGF-Trap was detected in the sera from mice that received AAV2-Null and NS. To evaluate the expression profile of AAV2-VEGF-Trap in normal mice after intravenous administration of AAV2-VEGF-Trap, the blood was obtained every week for 13 weeks. The expression of VEGF-Trap was sustained for 13 weeks (Fig. 3C) by adenoassociated virus mediated gene therapy. The result implied that a single intravenous administration of AAV2-VEGF-Trap can sustain the long-term expression of VEGF-Trap.

$A A V$-VEGF-Trapreduces the expression of angiogenesis-related factors. To analyze the relative levels of angiogenesis-related proteins, serum samples were detected via the Proteome Profiler Mouse Angiogenesis Array kit. Our results revealed that the expression of many angiogenic growth factors had been obviously reduced in mice treated with AAV2-VEGF-Trap than the control groups (Fig. 4, Table I), which suggested that the decreased tumor volumes and metastasis of AAV2-VEGF-Trap group may be caused by decreased expression of the angiogenesis-related proteins.

Toxicity of AAV2-VEGF-Trap. In general, the viruses have security issues. We examined the potential toxicity of AAV2VEGF-Trap by measuring changes in body weight every 3 days, and morphological analyses of major organs. There was no obvious difference in body weight between the experimental group and the control groups (Fig. 5A). Sections of heart, liver, spleen and kidney were stained with hematoxylin and eosin. Overall, no obvious or significant differences were detected in response to AAV2-VEGF-Trap and AAV2-Null compared with NS group (Fig. 5B).

\section{Discussion}

Angiogenesis plays a key role in tumor growth and metastasis. Tumor angiogenesis is regulated by a variety of active substances. Many angiogenic factors have been implicated in the regulation of the growth and metastasis of solid tumors. VEGF is a main angiogenic factor $(27,28)$, both in physiological or pathological processes it has important implications (29). In angiogenesis, tumor growth and metastasis, VEGF plays a critical role and is overexpressed in the majority of solid 
tumors, consistent with its role as a key mediator of tumor neoangiogenesis. Most tumors induce new vessel formation, suggesting that this neovascularization is required for their growth (30-32). Thus, blocking the formation of neovascularization is critical for inhibiting tumor growth. VEGF is an important and effective target for use against cancer, by binding and blocking VEGF to inhibit the growth and metastasis of tumors.

We have constructed a recombinant adeno-associated virus (AAV) expressing VEGF-Trap for the first time as shown both in vitro and in vivo models of angiogenesis. VEGF-Trap, a high-affinity soluble decoy receptor, comprises portions of the extracellular domains of both the second Ig domain of VEGFR1 with the third Ig domain of VEGFR2 fused to an Fc segment of human IgG. In clinical trials, VEGF-Trap has been shown to require repeated intravenous administration to maintain adequate therapy levels with the accompanying high cost, and the patient's resistance compared to a single administration. AAV vectors have not shown any danger and are very simple, so it can persist stably in transduced cells and achieve long-term transgene expression. Thus, in the present study, we chose recombinant AAV2 as gene delivery system to study the efficacy of VEGF-Trap in the mouse tumor model.

Transfected with AAV2-VEGF-Trap the cells led to expression of VEGF-Trap compared with the control, in vitro. Treatment of tumor-bearing mice with a single intravenous administration of $6 \times 10^{9} \mathrm{vg}$ of AAV2-VEGF-Trap caused a tumor volume reduction. In addition, lung metastases markedly decreased in both size and number in the 4T1 model. Analyzing tumor microvessels with CD31 immunohistochemistry showed an obvious reduction of microvessel density in AAV2-VEGF-Trap, compared with the NS and AAV2-Null groups. Furthermore, AAV2-VEGF-Trap increased the extent of apoptosis over that produced by the control groups. We demonstrated that injection of AAV2VEGF-Trap resulted in persistent high levels of VEGF-Trap in serum $(0,1,3,7,14,21$ and 28 days after administration of AAV2-VEGF-Trap). The normal female Balb/c mice with a single intravenous administration of $6 \times 10^{9} \mathrm{vg}$ of AAV2VEGF-Trap can be achieved obvious long-term expression for at least three months. No VEGF-Trap was detected in the injection of AAV2-Null. Through detecting the relative levels of angiogenesis-related proteins in the sera, the result showed obvious decrease not only of the concentration of VEGF in sera, but also the concentration of other angiogenic factor, such as aFGF, bFGF and angiopoietin-1.

Most preclinical and clinical studies of anti-VEGF agents have focused on targeting VEGF or its receptors. Several agents have shown promise in controlling tumor growth and lung metastasis, for example, VEGF-Trap. In previous studies, VEGF-Trap had minimum interactions with the extracellular matrix, and this property obviously accounts for its satisfactory pharmacokinetic profile, superior to soluble forms of VEGFR-1 to prevent VEGF from binding to its normal receptors by administering decoy VEGF receptor to block the VEGF signaling pathway (16,33-36). In previous studies, purified VEGF-Trap proteins were administered, with the major problem in pharmacotherapy of side effects of the constant VEGF-Trap administration and the limited half-life of VEGFTrap (37), requiring multiple injections. Gene therapy has advantages of maintaining sustained levels of VEGF-Trap in vivo, which enhanced antitumor efficacy. AAV vectors are widely used in preclinical and clinical gene therapy because of the low pathogenicity and toxicity, and sustained long-term expression of the target gene.

Taken together, AAV2-VEGF-Trap ultimately limits the blood supply and markedly increases tumor cell apoptosis. AAV2-mediated sustained expression of VEGF-Trap was safe and efficacious of gene therapy. This treatment dramatically reduces tumor burden and inhibits spontaneous metastasis via systematic effect of VEGF-Trap. In addition, long-term expression of a single administration of AAV-VEGF-Trap simultaneously reduces the expression of angiogenesis-related factors.

\section{Acknowledgements}

This study was funded by the Program for New Century Excellent Talents in University (NCET) (NCET-09-0575) and the National Key Basic Research Program (973 Program) of China (2010CB529900).

\section{References}

1. Sharma RA, Harris AL, Dalgleish AG, Steward WP and O'Byrne KJ: Angiogenesis as a biomarker and target in cancer chemoprevention. Lancet Oncol 2: 726-732, 2001.

2. Teo NB, Shoker BS, Martin L, Sloane JP and Holcombe C: Angiogenesis in pre-invasive cancers. Anticancer Res 22: 2061-2072, 2002.

3. Kieran MW and Billett A: Antiangiogenesis therapy. Current and future agents. Hematol Oncol Clin North Am 15: 835-851, viii, 2001.

4. Carmeliet P and Jain RK: Angiogenesis in cancer and other diseases. Nature 407: 249-257, 2000

5. Pour L, Hajek R, Buchler T, Maisnar V and Smolej L: Angiogenesis and antiangiogenic cancer therapy. Vnitr Lek 50: 930-938, 2004 (In Czech).

6. O'Reilly MS, Holmgren L, Shing Y, et al: Angiostatin: a novel angiogenesis inhibitor that mediates the suppression of metastases by a Lewis lung carcinoma. Cell 79: 315-328, 1994.

7. O'Reilly MS, Boehm T, Shing Y, et al: Endostatin: an endogenous inhibitor of angiogenesis and tumor growth. Cell 88: 277-285, 1997.

8. Moses MA and Langer R: A metalloproteinase inhibitor as an inhibitor of neovascularization. J Cell Biochem 47: 230-235, 1991.

9. Rastinejad F, Polverini PJ and Bouck NP: Regulation of the activity of a new inhibitor of angiogenesis by a cancer suppressor gene. Cell 56: 345-355, 1989.

10. Yancopoulos GD, Davis S, Gale NW, Rudge JS, Wiegand SJ and Holash J: Vascular-specific growth factors and blood vessel formation. Nature 407: 242-248, 2000.

11. Jain RK: Molecular regulation of vessel maturation. Nat Med 9: 685-693, 2003.

12. Leung DW, Cachianes G, Kuang WJ, Goeddel DV and Ferrara N: Vascular endothelial growth factor is a secreted angiogenic mitogen. Science 246: 1306-1309, 1989.

13. Kim KJ, Li B, Winer J, et al: Inhibition of vascular endothelial growth factor-induced angiogenesis suppresses tumour growth in vivo. Nature 362: 841-844, 1993.

14. Ferrara N and Davis-Smyth T: The biology of vascular endothelial growth factor. Endocr Rev 18: 4-25, 1997.

15. Millauer B, Shawver LK, Plate KH, Risau W and Ullrich A: Glioblastoma growth inhibited in vivo by a dominant-negative Flk-1 mutant. Nature 367: 576-579, 1994.

16. Holash J, Davis S, Papadopoulos N, et al: VEGF-Trap: a VEGF blocker with potent antitumor effects. Proc Natl Acad Sci USA 99: 11393-11398, 2002.

17. Kim ES, Serur A, Huang J, et al: Potent VEGF blockade causes regression of coopted vessels in a model of neuroblastoma. Proc Natl Acad Sci USA 99: 11399-11404, 2002. 
18. Gomez-Manzano C, Holash J, Fueyo J, et al: VEGF Trap induces antiglioma effect at different stages of disease. Neuro Oncol 10: 940-945, 2008

19. Zaiss AK and Muruve DA: Immunity to adeno-associated virus vectors in animals and humans: a continued challenge. Gene Ther 15: 808-816, 2008

20. Herzog RW, Yang EY, Couto LB, et al: Long-term correction of canine hemophilia B by gene transfer of blood coagulation factor IX mediated by adeno-associated viral vector. Nat Med 5: 56-63, 1999.

21. Wang L, Takabe K, Bidlingmaier SM, Ill CR and Verma IM: Sustained correction of bleeding disorder in hemophilia B mice by gene therapy. Proc Natl Acad Sci USA 96: 39063910, 1999.

22. Ponnazhagan S, Mahendra G, Kumar S, et al: Adeno-associated virus 2-mediated antiangiogenic cancer gene therapy: long-term efficacy of a vector encoding angiostatin and endostatin over vectors encoding a single factor. Cancer Res 64: 1781-1787, 2004.

23. Collins SA, Buhles A, Scallan MF, et al: AAV2-mediated in vivo immune gene therapy of solid tumours. Genet Vaccines Ther 8: $8,2010$.

24. Casanovas O, Hicklin DJ, Bergers G and Hanahan D: Drug resistance by evasion of antiangiogenic targeting of VEGF signaling in late-stage pancreatic islet tumors. Cancer Cell 8: 299-309, 2005.

25. Paley PJ, Staskus KA, Gebhard K, et al: Vascular endothelial growth factor expression in early stage ovarian carcinoma. Cancer 80: 98-106, 1997.

26. Heist RS, Zhai R, Liu G, et al: VEGF polymorphisms and survival in early-stage non-small cell lung cancer. J Clin Oncol 26: 856-862, 2008.
27. Johnson DE, Lee PL, Lu J and Williams LT: Diverse forms of a receptor for acidic and basic fibroblast growth factors. Mol Cell Biol 10: 4728-4736, 1990.

28. Basilico C and Moscatelli D: The FGF family of growth factors and oncogenes. Adv Cancer Res 59: 115-165, 1992.

29. Ferrara N: VEGF and the quest for tumour angiogenesis factors. Nat Rev Cancer 2: 795-803, 2002.

30. Ferrara $\mathrm{N}$ and Alitalo K: Clinical applications of angiogenic growth factors and their inhibitors. Nat Med 5: 1359-1364, 1999.

31. Folkman J: Angiogenesis in cancer, vascular, rheumatoid and other disease. Nat Med 1: 27-31, 1995.

32. Van Hinsbergh VW, Collen A and Koolwijk P: Angiogenesis and anti-angiogenesis: perspectives for the treatment of solid tumors. Ann Oncol 10 (Suppl 4): S60-S63, 1999.

33. Kuo CJ, Farnebo F, Yu EY, et al: Comparative evaluation of the antitumor activity of antiangiogenic proteins delivered by gene transfer. Proc Natl Acad Sci USA 98: 4605-4610, 2001.

34. Ferrara N, Chen H, Davis-Smyth T, et al: Vascular endothelial growth factor is essential for corpus luteum angiogenesis. Nat Med 4: 336-340, 1998.

35. Gerber HP, Vu TH, Ryan AM, Kowalski J, Werb Z and Ferrara N: VEGF couples hypertrophic cartilage remodeling, ossification and angiogenesis during endochondral bone formation. Nat Med 5: 623-628, 1999.

36. Gerber HP, Kowalski J, Sherman D, Eberhard DA and Ferrara N: Complete inhibition of rhabdomyosarcoma xenograft growth and neovascularization requires blockade of both tumor and host vascular endothelial growth factor. Cancer Res 60: 6253-6258, 2000.

37. Folkman J: Antiangiogenic gene therapy. Proc Natl Acad Sci USA 95: 9064-9066, 1998. 